Review Article

\title{
Reactive Oxygen Species and Nrf2: Functional and Transcriptional Regulators of Hematopoiesis
}

\author{
Linping Hu $\mathbb{D},{ }^{1,2,3}$ Yawen Zhang $\mathbb{D},^{1,2,3}$ Weimin Miao, ${ }^{1,2,3}$ and Tao Cheng $\mathbb{D}$ 1,2,3,4,5 \\ ${ }^{1}$ State Key Laboratory of Experimental Hematology, China \\ ${ }^{2}$ National Clinical Research Center for Blood Diseases, Institute of Hematology \& Blood Diseases Hospital, China \\ ${ }^{3}$ Chinese Academy of Medical Sciences \& Peking Union Medical College, Tianjin 300020, China \\ ${ }^{4}$ Center for Stem Cell Medicine, Chinese Academy of Medical Sciences, Tianjin, China \\ ${ }^{5}$ Department of Stem Cell \& Regenerative Medicine, Peking Union Medical College, Tianjin, China \\ Correspondence should be addressed to Linping Hu; hulinping@ihcams.ac.cn and Tao Cheng; chengtao@ihcams.ac.cn \\ Linping $\mathrm{Hu}$ and Yawen Zhang contributed equally to this work.
}

Received 13 July 2019; Revised 9 October 2019; Accepted 16 October 2019; Published 18 November 2019

Academic Editor: Joël R. Drevet

Copyright (c) 2019 Linping Hu et al. This is an open access article distributed under the Creative Commons Attribution License, which permits unrestricted use, distribution, and reproduction in any medium, provided the original work is properly cited.

\begin{abstract}
Hematopoietic stem cells (HSCs) are characterized by self-renewal and multilineage differentiation potentials. Although they play a central role in hematopoietic homeostasis and bone marrow (BM) transplantation, they are affected by multiple environmental factors in the BM. Here, we review the effects of reactive oxygen species (ROS) and Nrf2 on HSC function and BM transplantation. HSCs reside in the hypoxic microenvironment of BM, and ROS play an important role in HSPC regulation. Recently, an extraphysiologic oxygen shock/stress phenomenon was identified in human cord blood HSCs collected under ambient air conditions. Moreover, Nrf2 has been recently recognized as a master transcriptional factor that regulates multiple antioxidant enzymes. Since several years, the role of Nrf2 in hematopoiesis has been extensively studied, which has functional similarities of cellular oxygen sensor hypoxia-inducible factor-1 as transcriptional factors. Increasing evidence has revealed that abnormally elevated ROS production due to factors such as genetic defects, aging, and ionizing radiation unexceptionally resulted in lethal impairment of HSC function and hematopoiesis. Both experimental and clinical studies have identified elevated ROS levels as a major culprit of ineffective BM transplantation. Lastly, we discuss the possibility of using small molecule antioxidants, such as $\mathrm{N}$-acetyl cysteine, resveratrol, and curcumin, to augment HSC function and improve the therapeutic efficacy of BM transplantation. Further research on the function of ROS levels and improving the efficacy of BM transplantation may have a great potential for broad clinical applications of HSCs.
\end{abstract}

\section{Introduction to the Hematopoietic System}

Bone marrow (BM) transplantation has achieved great success in clinical practice and has saved numerous lives. Nevertheless, further improvements in its therapeutic efficacy are warranted. Various lineages of blood cells have been derived from common hematopoietic stem cells (HSCs) [1]. Hematopoiesis occurs in a hierarchical manner, with HSCs at the top as the cells of origin. They are able to self-renew and differentiate into various lineages of peripheral blood cells via hematopoietic progenitor cells [2-4]. HSCs have two basic properties: self-renewal and multipotent differentiation
$[4,5]$. However, molecular control underlying the stemness of these cells is still unclear and is thus a topic of interest.

HSC function is regulated by both intrinsic and extrinsic factors. Intrinsic factors arise from uniquely expressed signaling pathways in HSCs, whereas extrinsic factors arise from multiple factors in the microenvironment where HSCs reside, e.g., the BM niche [6-9]. Advances in single-cell and molecular technologies have led to a better understanding of the BM niche, both at homeostasis and under acute myeloid leukemia conditions $[7,10]$. BM niche represents a three-dimensional space comprising several types of components such as cells, blood vessels, extracellular matrices, 
cytokines, and adhesion molecules. Mesenchymal stem cells (MSCs), osteolineage cells, bone marrow-derived endothelial cells, chondrocytes, fibroblasts, and pericytes compose most niche cells, which interact with HSCs and regulate the function of HSCs [10]. Reactive oxygen species (ROS) represent another metabolic niche factor that has attracted increasing attention $[11,12]$. Moreover, Nrf2 has been recently recognized as a master transcriptional factor that regulates multiple antioxidant enzymes. Thus, in this article, we have summarized advancements in the assessment of the effects of ROS and Nrf2 on HSC function and BM transplantation.

\section{Sources of ROS and Cellular Redox Homeostasis}

Endogenous ROS are primarily derived from oxidative metabolism in the mitochondria, physiological metabolism processes, and inflammatory reactions. The examples of ROS include superoxide anion $\left(\mathrm{O}_{2}{ }^{-}\right)$, hydrogen peroxide $\left(\mathrm{H}_{2} \mathrm{O}_{2}\right)$, and hydroxyl ion $\left(\mathrm{OH}^{-}\right)$. Several hypotheses exist regarding the sources of ROS; however, mitochondria and membrane NADPH oxidase (NOX) are the two most recognized sources $[13,14]$. During cell proliferation, numerous biological macromolecules are involved in signal transduction and energy metabolism; ROS are formed as byproducts of these two processes. Current studies have revealed that ROS are not always harmful to cells; by contrast, ROS can serve as important signaling molecules [15-17]. ROS levels fluctuate during different cell cycle states of HSCs, affecting their motility, proliferation, differentiation, and repopulation potential. Also, elevated ROS levels in HSCs and MSCs promote HSC migration and mobilization [18]. Recently, Lapidot's group revealed that the oscillatory ROS levels in hematopoietic stem and progenitor cells (HSPCs) were driven by light and dark signals through different mechanisms. Those resulted in BM HSPC differentiation and replenishment with mature blood cells during the day and replenishment of the BM pool of stem and progenitor cells at night [19]. However, when ROS levels become abnormally high, HSCs may initiate a protective mechanism to shut down self-renewal functions. High ROS levels are known to cause cellular DNA damage and cell cycle arrest. Subsequently, DNA damage repair is initiated in affected cells. If the damage is successfully repaired, cells may continue to proliferate and differentiate. However, if the damage is too severe to be repaired, cells may undergo senescence or apoptosis [20]. Over the course of evolution, a complex defensive network has arisen to scavenge ROS to maintain redox balance in cells (Figure 1). Briefly, accumulated $\mathrm{O}_{2}{ }^{-}$ molecules in cells are first converted by superoxide dismutase (SOD) into $\mathrm{H}_{2} \mathrm{O}_{2}$. As $\mathrm{H}_{2} \mathrm{O}_{2}$ is toxic to cells, it is quickly converted into harmless water $\left(\mathrm{H}_{2} \mathrm{O}\right)$ in the presence of catalase or glutathione peroxidase (GPX1). However, if cells contain insufficient catalase and GPX1 to detoxify $\mathrm{H}_{2} \mathrm{O}_{2}$, the remaining $\mathrm{H}_{2} \mathrm{O}_{2}$ is converted into even more toxic $\mathrm{OH}^{-}$ions. $\mathrm{OH}^{-}$ ions can lead to destruction of cellular macromolecules including proteins, nuclear acids, and lipids [21]. Therefore, ROS detoxification requires a coordinated group of redox modulating enzymes. SOD appears to be a double-sided sword. On the one hand, it is a well-known antioxidant; on the other hand, it is a potent prooxidant, depending on the presence of other antioxidant enzymes such as catalase and GPX1 in cells. Nrf2 was recently discovered as a global regulator of cellular antioxidant defense [22]. Till date, a variety of ROS-detoxifying enzymes have been found to be regulated by the Nrf2/Keap1 pathway as downstream effectors, including SOD, catalase, GPX1, and thioredoxin.

\section{Role of Hypoxic Environment in Maintaining Stemness of HSCs}

It is generally believed that HSCs reside in the hypoxic microenvironment of BM. The oxygen tension of BM was quite low (<32 mm Hg) and heterogenous measured by two-photon phosphorescence lifetime microscopy. The highest oxygen tension in the BM was found next to the endosteum and arteries, while the lowest tension was next to the sinusoids [23]. However, the ROS levels of HSCs which resided in endosteal and arterial areas with high oxygen tension were low, while the ROS levels of HSCs which resided in sinusoids were high unless they were found next to a megakaryocyte who maintained them at low ROS state. The aforementioned phenomenon might be accounted for different blood vessel permeability (less in endosteal and arterial, more in sinusoids) and signals provided by the microenvironment [24]. Manipulation of BM vascular permeability shifted ROS levels in HSCs. Circulating HSCs or HSCs exposed to blood plasma were shown to have the highest ROS levels, increased ability of migration and differentiation, and decreased long-term repopulation potential [24]. Interestingly, when BM or cord blood (CB) is collected or treated in ambient air, HSCs quickly differentiate and significantly decrease in number. The phenomenon was recently reported by Mantel's group, who termed it extra physiologic oxygen shock/stress (EPHOSS) [25]. EPHOSS appears to be associated with elevated ROS. It is mediated by the p53-cyclophilinmitochondrial permeability transition pore (MPTP) signal axis, which involves hypoxia-inducible factor- $1 \alpha$ (HIF- $1 \alpha$ ) and $m i R 210$. To avoid EPHOSS, low oxygen tension (3\%) is recommended for HSC collection and handling. An alternative strategy is to collect HSCs under normal oxygen tension but to pretreat the collection tube with cyclosporine A (CSA). The compound CSA, which works on the MPTP axis, can be used to inhibit ROS production and thus improve CB-HSC collection $[25,26]$. Moreover, under AML conditions, increased BM vascular permeability resulted in reduced BM oxygen tension, while residual HSCs showed higher levels of ROS and reduced stemness and were mobilized to the periphery [27]. Additionally, it was confirmed that AML cells remodeled endosteal regions and have elevated ROS levels through the proinflammatory and vascular endothelial growth factor signaling pathway [28]. The numbers of healthy hematopoietic cells transmigrating across endothelial cells were increased in leukemic mice and may be associated with the elevated ROS levels [28]. In summary, the ROS levels of HSC were affected by various factors, including location, alternations of vessel permeability, or whether it is under stress. 


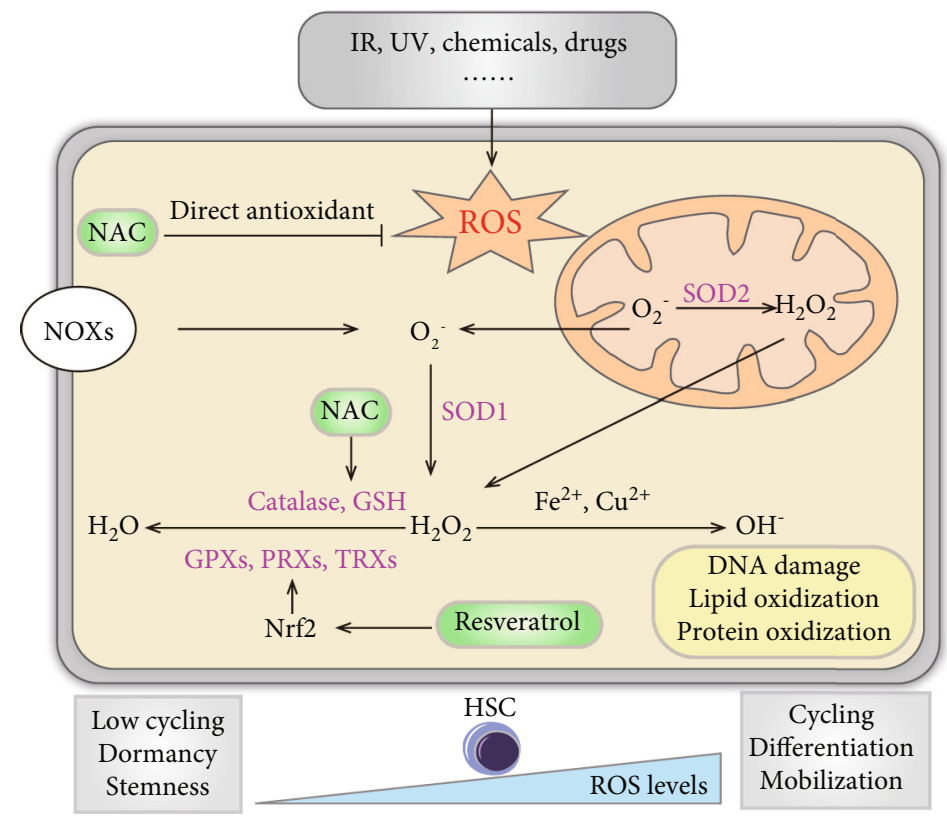

FIGURE 1: Sources of ROS and regulation cellular redox homeostasis in hematopoiesis. Ionizing radiation (IR), ultraviolet (UV), chemical drugs, etc. result in exogenous elevated ROS levels. Accumulated $\mathrm{O}^{-}$molecules by mitochondria and membrane NADPH oxidase (NOX) in cells are first converted by superoxide dismutase (SOD) into $\mathrm{H}_{2} \mathrm{O}_{2} \cdot \mathrm{H}_{2} \mathrm{O}_{2}$ quickly converts into harmless water $\left(\mathrm{H}_{2} \mathrm{O}\right)$ in the presence of catalase, glutathione (GSH), glutathione peroxidase (GPX), peroxiredoxin (PRX), and thioredoxin (TRX). If cells are insufficient to detoxify $\mathrm{H}_{2} \mathrm{O}_{2}$, the remaining $\mathrm{H}_{2} \mathrm{O}_{2}$ is converted into even more toxic $\mathrm{OH}^{-}$ions. $\mathrm{OH}^{-}$ions can lead to destruction of cellular macromolecules including proteins, nuclear acids, and lipids. N-acetyl cysteine (NAC) and resveratrol are two small molecule antioxidants which reduce ROS levels. In hematopoiesis, dormant, quiescent HSCs are characterized by low ROS levels. Elevated ROS levels in HSCs enhance cycling and promote differentiation and mobilization.

Moreover, ROS play an important role in HSC regulation. On the one hand, low ROS levels are essential for quiescent HSCs to maintain their stemness; on the other hand, a physiological level of ROS is needed to promote HSC proliferation and differentiation [29]. However, abnormally elevated ROS can result in DNA damage of the $\mathrm{CD} 34^{+}$hematopoietic fraction, reduced colony formation, and impaired proliferative capacity [30]. Recently, a novel mechanism of HSPC regulation was verified. A signaling network of p190B RhoGAP-ROS-TGF- $\beta$-p $38^{\text {MAPK }}$ balances HSPC selfrenewal and differentiation [31]. This finding further confirms that ROS play an important role in HSC regulation.

\section{Role of Nrf2 in Redox Regulation of HSCs and Microenvironment}

Nrf2 is well established as a master transcriptional factor regulating various antioxidant enzymes. The role of Nrf2 in hematopoiesis has been extensively studied in recent years. ROS levels are abnormally increased in the liver and kidney cells of Nrf2 null mice, suggesting that Nrf2 is essential for metabolizing ROS [32]. Kato et al. studied the relationship between radiation sensitivity and NRF2-regulated gene expression profiles using CB-HSCs. They performed X-ray irradiation of $\mathrm{CD} 34^{+}$human $\mathrm{CB}-\mathrm{HSCs}$ and then examined the downstream antioxidant genes regulated by NRF2. The results showed that after HSCs were irradiated at a dose of $2 \mathrm{~Gy}$, the number of colony-forming cells (CFCs) decreased significantly to $20 \%$ of that in the control group. Addition- ally, multiple antioxidant genes regulated by NRF2-such as NADPH dehydrogenase quinone 1 (NQO1), glutathione reductase (GSR), thioredoxin reductase 1 (TXNRD1), heme oxygenase 1 (HO-1), and ferritin heavy chain 1 (FTH1)were upregulated after irradiation. Although the research is preliminary, the data revealed a critical role of NRF2 in defensive reactions against ionizing radiation (IR) [33]. Studies using Nrf2 knockout mice have shown that $\mathrm{Nrf2}^{-/-}$HSCs are severely defective and more sensitive to oxidative stress. Surprisingly, ROS levels of Nrf2 ${ }^{-/-}$HSCs are at basal levels and the defects of HSCs cannot be restored by treatment with $\mathrm{N}$-acetyl cysteine (NAC). This suggests that induced ROS is not the main cause of HSC defect. However, granulocyte colony-stimulating factor (G-CSF) treatment could rescue HSC function after exogenous oxidative stress despite causing elevated ROS levels which indicates involvement of a cytokine defect in $\mathrm{Nrf2}^{-/-}$HSC. High ROS is actually harmful to cells, but in some cases, normal ROS levels cannot represent the undisturbed HSC function; new markers and strategies are needed to measure oxidative stress and cellular damage [34]. In a separate study of Nrf2-deficient mice, significant expansion of HSPCs was found to occur at the expense of HSC quiescence and self-renewal. Since the phenotype of Nrf2-deficient HSCs is somewhat similar to that of forkhead O- (FOXO-) deficient HSCs, the author speculated that Nrf2 functions in parallel to FOXO proteins as a downstream target of the PI3K-Akt pathway and that this mechanism warrants further investigation [35]. Kim et al. [36] demonstrated that activation of 
Nrf2 signaling, either by genetic deletion of Keap1 or by treatment with the small molecule activator 2-trifluoromethyl-2' methoxychalone (TMC), augments HSPC function and attenuates IR-induced BM suppression and mortality. TMC administration results in activation of Notch signaling in HSPCs and leads to enhanced HSPC expansion [36, 37]. Recently, Murakami et al. [38] demonstrated that Nrf2 drives cell cycle entry and differentiation of LT-HSCs at the expense of their quiescence and maintenance through Keap1. Moreover, the Nrf2 pathway is involved in mediating abnormal hematopoiesis (such as benzene-induced hematotoxicity) in response to oxidative stress [39]. In summary, Nrf2 function vitally in redox regulation of HSCs.

In addition to HSCs, Nrf2 also plays a global role in regulating oxidative stress in niche cells such as MSC, megakaryocytes, Schwann cells, and T cells. Nrf2 known down in MSCs resulted in elevated ROS levels, reduced target antioxidant gene expression, and depressed cell survival. Conversely, reexpression of Nrf2 rescued the increased oxidative stress and restored the in vitro and in vivo viability of MSCs [40]. Nrf2 was also proved to maintain stem cell properties and the osteogenesis process in MSCs [41]. Moreover, by competing with Nrf2, NF-E2 p45 regulated cytoprotective genes and promoted elevated ROS levels to enhance megakaryocytic maturation [42]. Also, Nrf2 ${ }^{-/-}$mice showed impaired functional recovery after peripheral nerve injury [43]. Slight oxidative stress activated Nrf2, enhanced the expression of antioxidant enzymes such as catalase and $\mathrm{HO}-1$, promoted neurons to generate glutathione (GSH), and thus ultimately protected sensory neurons and Schwann cells from oxidative damage [44]. A critical role of Nrf2 in T cell function has been revealed by Brink's group. Expression and nuclear translocation of Nrf2 were significantly increased after $\mathrm{T}$ cell activation in vitro and $\mathrm{CD} 4^{+}$donorderived T cells. Nrf2 $2^{-/-}$donor-derived T cells induced less acute graft-versus-host disease through increased $\mathrm{Helios}^{+}$ donor regulatory $\mathrm{T}$ cells. In detail, $\mathrm{Nrf} 2$ reduced $\mathrm{CD} 25$ expression and moderated IL-2 signaling resulting in decreasing Helios and Foxp3 expression [45]. In conclusion, Nrf2 protects niche cells from oxidative stress and creates a favorable microenvironment for HSCs.

As a well-established global regulator of redox homeostasis, Nrf2 plays an essential role in other stem cell types, including embryonic stem cells (ESCs), MSCs, and cancer stem cells (CSCs). For example, Nrf2 is involved in the control of self-renewal properties and pluripotency in human ESCs [46] and is essential for maintaining quiescence and differentiation in adult stem cells including Drosophila intestinal stem cells [47], human airway basal stem cells [48], and human MSCs [40]. Nrf2 also contributes to maintenance of quiescence, survival, and stress resistance in CSCs $[49,50]$.

\section{Genetic Defects Unveiling the Signaling Pathways for Redox Regulation in HSCs}

Studies using genetically deficient mouse models have shed light on the signaling pathways that mediate ROS-induced impairment in HSCs $[13,21]$. For example, ATM mutation has been shown to lead to ataxia telangiectasia, an autosomal recessive genetic disorder in humans. Aged $\mathrm{Atm}^{-/-}$ mice display progressive $\mathrm{BM}$ failure due to severe defects in HSC function. These HSC defects are associated with high levels of ROS, since treatment with the antioxidant NAC significantly improves HSC reconstitution capacity in $\mathrm{Atm}^{-/-}$mice [51]. Mechanistic studies have further revealed that high ROS levels in $\mathrm{Atm}^{-/-}$mice activate the p38MAPK pathway, contributing to HSC exhaustion. Treatment with a p38 inhibitor restores ROS-induced defects in HSC reconstitution ability and in the maintenance of HSC quiescence [52].

The FoxO family of transcription factors plays active roles in regulating genes involved in cell growth, proliferation, differentiation, and metabolism. The family comprises four members, namely, FoxO1, FoxO3, FoxO4, and FoxO6, present in mammalian cells. Studies [53] have shown that FoxO transcription factors are essential for controlling redox homeostasis of HSCs. Gilliland's group created conditional gene deletions for Foxo1, Foxo3a, and Foxo4. In FoxO triple-deletion mice, the number of hematopoietic LSK (Lin-Scal-1+C-Kit+) cells was markedly decreased. Moreover, HSCs from FoxO-deficient mice had a poor long-term repopulating capacity due to increased differentiation and apoptosis [53]. Notably, the HSC defects were associated with high ROS levels, which resulted in DNA damage [54], and the FoxO HSC phenotype could be reversed by treatment with NAC $[55,56]$. Although FoxO family members have both distinct and overlapping functions, a single FoxO3a deletion can result in a similar defect in HSC long-term repopulating activities associated with high ROS levels. High ROS levels have been shown to lead to phosphorylation of p38MAPK in FoxO3a-deficient cells, which activates the cellular senescence-related genes $p 16^{\text {Ink4a }}$ and $p 19^{\text {Arf }}$ and consequently results in loss of HSC function. Additionally, the HSC defects could be repaired by treatment with p38 inhibitors or the antioxidant NAC [57]. Although several upstream signaling pathways may be involved, PI3K/Akt is mostly recognized for its negative regulation of FoxO transcriptional factors [58]. Furthermore, the microRNA-212/132 cluster is known to regulate expression of $\mathrm{FoxO} 3$, and its overexpression or knockout can lead to hematopoietic defects [59].

Fanconi anemia (FA) is a genetic disorder characterized by progressive BM failure, congenital defects, and predisposition to cancer. FA is caused by a defect in any of the 16 FA genes (FANCA-Q), all of which are involved in the DNA repair pathway. FANCD2 is downstream of a large nuclear complex composed of multiple FA proteins. Fancd2-deficient mice have a variety of HSC defects including loss of quiescence, abnormal cell cycle, and compromised function. Li's group reported an oxidative stressspecific interaction between FANCD2 and FOXO3a: a novel pathway in which FANCD2 and FOXO3a colocalize and cooperate was found to be involved in the cellular oxidative stress response [60]. To study the impact of this pathway on hematopoiesis, the authors constructed Fancd 2 and Foxo3a double-knockout (DKO) mice. Deletion of both Fancd 2 and Foxo3a resulted in an initial expansion 
followed by progressive reduction of HSPCs. DKO HSCs showed dramatic reduction in hematopoietic reconstitution ability in transplantation experiments. Moreover, Fancd2 was shown to be required for nuclear retention of Foxo3a, Fancd2 deficiency kept Foxo3a localized in the cytoplasm of HSCs, and reexpression of Fancd2 rescued Foxo3a nuclear localization [61].

Besides genetic regulation, epigenetic regulation from noncoding RNA is also involved in redox regulation of hematopoiesis. Qian et al. reported that the mammalian imprinted Dlk1-Gtl2 locus plays an important role in sustaining long-term HSC properties [62]. Mechanistic studies have demonstrated that the miRNA megacluster within the Dlk1-Gtl2 locus inhibits the entire PI3K-mTOR pathway, which in turn suppresses mitochondrial biogenesis and reduces ROS production [62].

\section{Excessive ROS Production in Hematopoietic Tissue during Aging}

It has long been observed that HSCs from aging mice have low homing and engraftment efficiencies [63] and poor therapeutic efficacy [64]. Although it is taken for granted that aging compromises HSC function [65], the precise molecular mechanism is yet to be elucidated. Elevated ROS levels have been speculated as one of the causes of HSC defects in aging mice. Meyrelles et al. conducted comparison experiments among the young-, intermediate, and old-age groups (younger than 2, 12, and 24 months, respectively) of C57BL/6J mice. Their data showed that intracellular ROS levels in HSCs were significantly higher in the old-age group than in the young-age group. Moreover, the ROS sources were different. Mitochondrial and NOX sources were common in all three groups while the CYP450 source was observed in the intermediate- and old-age groups; by contrast, the xanthine oxidase source was only observed in the old-age group. In old-age mice, high levels of ROS were associated with more differentiated HSCs. "Aging" HSCs showed high levels of DNA damage and high rates of senescence and/or apoptosis [66]. Khatri et al. found that, in aged mice, BM stromal cells accumulate more ROS than do HSCs, which considerably reduces cellularity in the BM. Notably, elevated ROS levels compromise the ability of BM stromal cells to support HSC reconstitution in aged mice [67]. Treatment with the antioxidant curcumin has been shown to significantly improve hematopoietic reconstitution in aged mice. In addition, favorable effects of the ginsenoside $\mathrm{Rg} 1$ [68] and angelica sinensis polysaccharide (ASP) [69] on HSPCs via suppression of ROS in an induced aging mouse model have been reported. In another study, aged microenvironment was shown to contribute to the aging of HSCs. Aged endothelial cells showed increased ROS levels and promoted myeloid-biased hematopoiesis and HSC functional decline. Young ECs could protect the aged BM microenvironment and rejuvenate the aged hematopoietic system. And young ECs, which have lower ROS levels, may act as a supportive clinical cellular therapy [70]. Taken together, excess production of ROS by HSCs and niche cells during aging accounted for HSC defects. Hence, preventing excessive ROS production could be a feasible method to promote rejuvenation of aged hematopoietic tissue.

\section{Elevated Cellular ROS Levels Induced by Exogenous Insults}

The most studied exogenous insult is IR [68]. IR exposure resulting from nuclear accidents, terrorist attacks, or radiation therapy represents a major medical concern. BM is one of the most susceptible tissues to IR. IR is known to cause acute and long-term BM suppression, and patients with BM impairments after IR exposure may experience severe hematological symptoms or even death. ROS have been confirmed as the major cause of long-term BM suppression after irradiation. High levels of ROS induced by IR greatly impair HSC self-renewal and induce senescence, ultimately leading to HSC exhaustion. Further research has shown that ROS-induced HSC senescence is mediated by redoxdependent activation of the $\mathrm{p} 38^{\mathrm{MAPK}}-\mathrm{p} 16^{\mathrm{ink} 4 \mathrm{a}}$ pathway [71]. Recently, low-dose irradiation, which was previously linked to leukemia, was proven to promote persistent oxidative stress and decrease self-renewal of HSCs [72]. Importantly, treatment with the antioxidants resveratrol [73], isorhapontigenin, heyneanol-A [74], metformin [75], chlorophyllin [76], 3,3' -diindolylmethane [77], or methoxytryptamine- $\alpha$-lipoic acid [78] can effectively reduce IRinduced ROS, thereby preventing or mitigating IRinduced hematopoietic dysfunctions.

Besides IR, various environmental toxins can induce cellular ROS production $[79,80]$. For example, lead is a heavy metal environmental toxin that can impair various organs and tissues, including the hematopoietic system. Elevated cellular ROS levels are regarded as a major mechanism of lead toxicity. Liu et al. reported that lead-exposed rats have developmental defects in myeloid and lymphoid lineages. Indeed, lead exposure results in senescence/apoptosis and functional loss of HSCs, all of which are associated with mitochondrial defects and increased ROS generation [80]. Treatment with the antioxidant vitamin $\mathrm{C}$ can alleviate the functional decrease in lead-exposed HSCs [80]. The adverse effect of iron overload on HSCs offers further evidence. Iron overload due to congenital hemochromatosis or repeated blood transfusions can lead to BM failure and parenchymal organ disorders. Lu et al. [81] studied the effect of iron overload on hematopoiesis in patients with transfusional iron overload. In vitro experiments have demonstrated that iron overload results in apoptosis, cell cycle arrest, and compromised function of BM-derived mononuclear cells and umbilical cord-derived MSCs, which are correlated with elevated ROS levels and activated p38MAPK and p53. Importantly, NAC or deferoxamine (DFO) can mitigate iron overloadinduced HSC impairments [81]. Using a mouse model, Chai et al. [82] demonstrated that iron overload significantly decreased the proportion and clonogenic function of murine HSPCs. The impairments were associated with elevated ROS levels and could be corrected by treatment with NAC or 
deferasirox (DFX). These treatments remarkably reduced ROS levels by suppressing NOX4 and p38 4 APK and improved hematopoietic reconstitution of HSCs impaired by iron overload after transplantation [82].

\section{Adverse Effects of ROS on BM Transplantation}

Numerous experiments with mouse models have indicated that elevated ROS levels are a major negative factor for BM transplantation. We reported that recipient mice produce high levels of ROS after total body irradiation (TBI) (before transplantation), which can elicit a type of "bystander effect" on transplanted HSCs [83]. The affected HSCs then undergo proliferation-independent exhaustion, which is marked by a dramatic decrease in the expression of the c-kit gene and is correlated with elevated ROS levels. Again, administration of NAC can reverse the harmful effects and significantly improve transplant engraftment. Another study [84] by our group showed that the baseline expression levels of two major ROS-metabolizing enzymes, namely, MnSOD and catalase, are very low in HSCs. Injection of MnSOD plasmid liposomes into recipient mice before TBI markedly reduced ROS levels in the $\mathrm{BM}$, which significantly improved HSC transplant engraftment. The authors [84] also successfully constructed a retroviral vector to overexpress MnSOD or catalase in mouse HSCs. Overexpressed catalase had a marked positive effect on long-term reconstitution of transplanted HSCs, and this effect was further augmented after another sublethal irradiation challenge in the transplanted mice. However, overexpressed MnSOD had a less beneficial effect than catalase on the reconstitution of transplanted HSCs.

Clinical investigations have confirmed our finding of the negative effects of ROS on BM transplantation in mouse models [83-85]. Using a prospective paired study, Huang's group found that an elevated ROS level was a major risk factor for "poor graft function (PGF)" after allogeneic BM transplantation. Mechanism studies have further revealed that ROS cause DNA damage, HSC apoptosis (especially CD34+CD38- HSC), HSC exhaustion, and defective CFU formation. Increased levels of $\mathrm{p} 53$, p21, caspase-3, and caspase-9 (but not p38) have also been detected in ROS-affected HSCs [86].

\section{Potential Application of Small Molecule Antioxidants in BM Transplantation}

As discussed earlier, mobilized peripheral HSCs showed elevated ROS levels due to the G-CSF treatment [18], exposure to blood plasma elements [24], and EPHOSS [25]. Thus, reducing ROS levels through ex vivo small molecule antioxidant treatment, low oxygen tension for HSC collection, and exogenous Nrf2 activation could be feasible methods to improve engraftment efficiency via maintaining stemness of HSC. In addition, in vivo reducing ROS levels post transplantation in the BM microenvironment could promote better niche function for an increase in hematopoietic engraftment.
The most widely used small molecule antioxidant in hematopoiesis research is likely NAC, a medication used to treat paracetamol overdose, mucolytics, hemorrhagic cystitis, and obstructive lung disease, among others. It is also sold as a dietary supplement for its antioxidant effects. NAC is a precursor of L-cysteine and reduced GSH which functions as the antioxidant in a direct and indirect way. NAC directly scavenges $\mathrm{O}_{2}{ }^{-}, \mathrm{H}_{2} \mathrm{O}_{2}, \mathrm{OH}^{-}$, and other free radicals through the $-\mathrm{SH}$ thiol group to exert direct antioxidant activity [87]. Also, NAC acts as an indirect antioxidant by increasing the intracellular GSH concentration to reduce redox stress [88] (Figure 1). Numerous studies to date have used NAC to improve the therapeutic effects of BM transplantation. NAC has also been used to assess the impact of ROS on HSCs, restore genetic defects in the redox pathway, overcome the bystander effect caused by IR, and improve $\mathrm{BM}$ transplantation efficiencies in immunodeficient mice (NOD/SCID) [51, 55, 81-83]. NOD/SCID mice are commonly used for in vivo transplantation studies of human HSCs; however, the engraftment efficiency is unsatisfactory. Our group [85] detected higher levels of ROS in the BM of NOD/SCID mice relative to other normal mouse strains (C57BL/6 and $\mathrm{BALB} / \mathrm{C})$. Administration of $\mathrm{NAC}$ was found to reduce ROS levels in the BM of NOD/SCID mice. Moreover, NAC treatment significantly improved human HSC engraftment and multilineage hematopoietic differentiation in these mice. Compared with control mice, NAC-treated recipient mice had a 10.8 -fold increase in hematopoietic engraftment in the injected tibiae [85]. Recently, NAC has been used in clinical trials. A pilot study by Kong's group [89] reported an overall response of NAC treatment in 7 of 10 prolonged isolated thrombocytopenia (PT) patients without significant side effects. Furthermore, posttreatment platelet counts in the treated group of PT patients were significantly increased compared to pretreatment. Another study demonstrated that the occurrences of PGF and PT were significantly reduced, as were the ROS levels, in an $\mathrm{EC}<0.1 \%$ patient group that received preventative NAC [90].

After NAC, resveratrol is perhaps the second most investigated antioxidant in HSC studies. The direct antioxidant effects of resveratrol as the ROS scavenger are not strong, while resveratrol can act as a gene regulator of the redox system which enhances the transcriptional activity of Nrf2 and Sirt1 [91]. Resveratrol has been used to augment ex vivo HSC expansion, improve BM transplantation efficiency, and-in particular-protect HSCs from IR. Human CB-HSCs are an important source for HSC transplants. Since the number of CB-HSCs is low, it is necessary to perform in vitro amplification before transplantation. Many small molecules including antioxidants have been tested for use in CB-HSCs ex vivo expansion. Schiedlmeier's group reported that the antioxidant resveratrol, together with common cytokine combinations (including stem cell factor, thrombopoietin, Fms-related tyrosine kinase 3 ligand, and interleukin-6), significantly promoted CB-HSCs ex vivo expansion. Moreover, resveratrol has been shown to potently support the multilineage 
reconstitution of human CB-HSCs in NSG (NOD/SCI$\left.\mathrm{D} / \mathrm{IL} 2 \mathrm{r} \gamma^{-1-}\right)$ mice [92]. Rimmele et al. [93] reported that after mice were given resveratrol for three weeks, the number of functional HSCs significantly increased. In vivo experiments have shown that the function of multipotent progenitor cells is improved by resveratrol [93]. Meng's group reported that resveratrol can mitigate damage to HSCs caused by IR. Administration of resveratrol can also ameliorate acute BM symptoms and death rate and attenuate long-term HSC impairment caused by IR by reducing ROS levels [73]. Heyneanol appears to have even greater therapeutic efficacy than resveratrol and isorhapontigenin [74]. Besides NAC and resveratrol, antioxidants including curcumin [67], TMC [36], alendronate [94], vitamin A, and vitamin $\mathrm{E}$ have been used during $\mathrm{BM}$ transplantation or to treat ROS-related hematological diseases. How to select the right antioxidant for a particular patient needs to be defined from future clinical trials in the transplant patients with different antioxidants. Efficacy of a specific antioxidant for BM transplantation will largely depend on the preconditioning regimen protocol and other important factors such as disease type/stages and donor cell types/doses. The interactions of different chemotherapy drugs and antioxidants still need to be taken into consideration for choosing the right antioxidant. Furthermore, new agents targeting a specific microenvironment or cells may be also considered a combinational therapy in the future.

\section{Concluding Remarks}

In summary, primitive HSCs typically reside in the hypoxic environmental niche in the BM. Although physiological low levels of ROS are necessary to maintain functional HSCs in hemostasis, aberrantly elevated ROS levels due to intrinsic or extrinsic insults may cause significant defects and ultimately exhaustion of HSCs. In fact, high ROS levels are a known risk factor for poor BM transplantation in experimental and clinical settings. Several small molecule antioxidants have been tested to reduce ROS levels and improve the efficacy of BM transplantation, demonstrating utility in clinical stem cell transplantation.

In the future, in situ measurement of ROS levels and single cell metabolomics could give us a new perspective of measuring ROS variations in three-dimensional space and detecting the heterogeneity of HSPCs. Moreover, considering the largely unsuccessful result of antioxidants in cancer prevention [95], more clinical trials involving small molecule antioxidants need to be tested in application of $\mathrm{BM}$ transplantation. Also, the transcriptional factor Nrf2 is important in regulating redox response elements and serves as an antioxidant to reduce ROS levels. More approaches that target Nrf2 for improving efficiency of BM transplantation could be the future direction. Nonetheless, the recent Nobel-winning work on HIF- $1 \alpha$ which mediated regulation in cellular oxygen sensing has some functional similarities with $\mathrm{Nrf} 2$ as transcriptional factors. Briefly, HIF- $1 \alpha$ regulates variations in oxygen partial pressure and Nrf2 functions in redox regulation. More and more potential drugs increasing HIF-1 function [96, 97] would assist in disease treatments as well as BM transplantation. Also, it will likely fuel the future studies on the roles of ROS in hematopoiesis as well as the clinical applications of antioxidants for stem cell transplant patients.

\section{Abbreviations}

HSCs: Hematopoietic stem cells

BM: $\quad$ Bone marrow

ROS: $\quad$ Reactive oxygen species

EPHOSS: Extra physiologic oxygen shock/stress

$\mathrm{O}_{2}^{-}: \quad$ Superoxide anion

$\mathrm{H}_{2} \mathrm{O}_{2}$ : Hydrogen peroxide

$\mathrm{OH}^{-}: \quad$ Hydroxyl ion

NOX: NADPH oxidase

SOD: $\quad$ Superoxide dismutase

GPX1: Glutathione peroxidase

MAPK: $\quad$ p38 mitogen-activated protein kinase

mTOR: Mammalian target of rapamycin

HSPCs: Hematopoietic stem and progenitor cells

CB: $\quad$ Cord blood

MPTP: p53-cyclophilin-mitochondrial permeability transition pore

HIF- $1 \alpha$ : Hypoxia-inducible factor- $1 \alpha$

CSA: Cyclosporine A

CFCs: Colony-forming cells

NQO1: NADPH dehydrogenase quinone 1

GSR: Glutathione reductase

TXNRD1: Thioredoxin reductase 1

HO-1: Heme oxygenase 1

FTH1: $\quad$ Ferritin heavy chain 1

IR: Ionizing radiation

NAC: N-acetyl cysteine

G-CSF: Granulocyte colony-stimulating factor

FOXO: Forkhead O

TMC: $\quad$ 2-trifluoromethyl-2' ${ }^{\prime}$-methoxychalone

GSH: Glutathione

ESCs: Embryonic stem cells

MSCs: Mesenchymal stem cells

CSCs: Cancer stem cells

LSK: $\quad$ Lin-Scal-1+C-Kit+

FA: $\quad$ Fanconi anemia

ASP: Angelica sinensis polysaccharide

DFO: Deferoxamine

DFX: Deferasirox

TBI: Total body irradiation

PGF: $\quad$ Poor graft function

PT: $\quad$ Prolonged isolated thrombocytopenia.

\section{Conflicts of Interest}

The authors declare that there is no conflict of interest regarding the publication of this paper.

\section{Authors' Contributions}

LH and YZ wrote the manuscripts. TC and WM gave suggestions and gave revisions. All authors contributed to the 
writing of the review. All authors read and approved the final manuscript. Linping $\mathrm{Hu}$ and Yawen Zhang contributed equally to this work.

\section{Acknowledgments}

This work was supported by grants from the Ministry of Science and Technology of China (2016YFA0100600, 2017YFA0103400, and 2015CB964400), the National Natural Science Foundation of China $(81400150,81421002$, 81890990, 81861148029, 81730006, 81970104, and 81922002), the PUMC Youth Fund and the Fundamental Research Funds for the Central Universities (3332016090), the CAMS Initiative for Innovative Medicine (2016-I2M-1017, 2017-I2M-3-009, and 2019-I2M-1-006), the Tianjin Science and Technology Commission (14ZCDZSY00176), the Jiangsu Science and Technology Commission (BC2015057), and the Nanjing municipality 321 talent program.

\section{References}

[1] M. A. Goodell, K. Brose, G. Paradis, A. S. Conner, and R. C. Mulligan, "Isolation and functional properties of murine hematopoietic stem cells that are replicating in vivo," Journal of Experimental Medicine, vol. 183, no. 4, pp. 1797-1806, 1996.

[2] M. Osawa, K. Hanada, H. Hamada, and H. Nakauchi, "Longterm lymphohematopoietic reconstitution by a single CD34low/negative hematopoietic stem cell," Science, vol. 273, no. 5272, pp. 242-245, 1996.

[3] I. L. Weissman, D. J. Anderson, and F. Gage, "Stem and progenitor cells: origins, phenotypes, lineage commitments, and transdifferentiations," Annual Review of Cell and Developmental Biology, vol. 17, pp. 387-403, 2001.

[4] E. Laurenti and B. Gottgens, "From haematopoietic stem cells to complex differentiation landscapes," Nature, vol. 553, no. 7689, pp. 418-426, 2018.

[5] A. Wilson, E. Laurenti, G. Oser et al., "Hematopoietic stem cells reversibly switch from dormancy to self-renewal during homeostasis and repair," Cell, vol. 135, no. 6, pp. 1118-1129, 2008.

[6] F. Arai and T. Suda, "Maintenance of quiescent hematopoietic stem cells in the osteoblastic niche," Annals of the New York Academy of Sciences, vol. 1106, pp. 41-53, 2007.

[7] A. N. Tikhonova, I. Dolgalev, H. Hu et al., "The bone marrow microenvironment at single-cell resolution," Nature, vol. 569, no. 7755, pp. 222-228, 2019.

[8] S. Pinho and P. S. Frenette, "Haematopoietic stem cell activity and interactions with the niche," Nature Reviews Molecular Cell Biology, vol. 20, no. 5, pp. 303-320, 2019.

[9] A. Wilson, E. Laurenti, and A. Trumpp, "Balancing dormant and self-renewing hematopoietic stem cells," Current Opinion in Genetics \& Development, vol. 19, no. 5, pp. 461-468, 2009.

[10] N. Baryawno, D. Przybylski, M. S. Kowalczyk et al., "A cellular taxonomy of the bone marrow stroma in homeostasis and leukemia," Cell, vol. 177, no. 7, pp. 1915-1932.e16, 2019.

[11] K. Ito, K. Takubo, F. Arai et al., "Regulation of reactive oxygen species by Atm is essential for proper response to DNA double-strand breaks in lymphocytes," The Journal of Immunology, vol. 178, no. 1, pp. 103-110, 2007.
[12] T. Suda, K. Takubo, and G. L. Semenza, "Metabolic regulation of hematopoietic stem cells in the hypoxic niche," Cell Stem Cell, vol. 9, no. 4, pp. 298-310, 2011.

[13] C. L. Bigarella, R. Liang, and S. Ghaffari, "Stem cells and the impact of ROS signaling," Development, vol. 141, no. 22, pp. 4206-4218, 2014.

[14] P. H. Willems, R. Rossignol, C. E. Dieteren, M. P. Murphy, and W. J. Koopman, "Redox homeostasis and mitochondrial dynamics," Cell Metabolism, vol. 22, no. 2, pp. 207-218, 2015.

[15] S. Nemoto, K. Takeda, Z. X. Yu, V. J. Ferrans, and T. Finkel, "Role for mitochondrial oxidants as regulators of cellular metabolism," Molecular and Cellular Biology, vol. 20, no. 19, pp. 7311-7318, 2000.

[16] L. A. Sena and N. S. Chandel, "Physiological roles of mitochondrial reactive oxygen species," Molecular Cell, vol. 48, no. 2, pp. 158-167, 2012.

[17] Y. Cao, Y. Fang, J. Cai et al., "ROS functions as an upstream trigger for autophagy to drive hematopoietic stem cell differentiation," Hematology, vol. 21, no. 10, pp. 613-618, 2016.

[18] A. Ludin, S. Gur-Cohen, K. Golan et al., "Reactive oxygen species regulate hematopoietic stem cell self-renewal, migration and development, as well as their bone marrow microenvironment," Antioxidants \& Redox Signaling, vol. 21, no. 11, pp. 1605-1619, 2014.

[19] K. Golan, A. Kumari, O. Kollet et al., "Daily onset of light and darkness differentially controls hematopoietic stem cell differentiation and maintenance," Cell Stem Cell, vol. 23, no. 4, pp. 572-585.e7, 2018.

[20] C. N. Weiss and K. Ito, "DNA damage response, redox status and hematopoiesis," Blood Cells Molecules and Diseases, vol. 52, no. 1, pp. 12-18, 2014.

[21] C. I. Kobayashi and T. Suda, "Regulation of reactive oxygen species in stem cells and cancer stem cells," Journal of Cellular Physiology, vol. 227, no. 2, pp. 421-430, 2012.

[22] G. M. DeNicola, F. A. Karreth, T. J. Humpton et al., "Oncogene-induced Nrf2 transcription promotes ROS detoxification and tumorigenesis," Nature, vol. 475, no. 7354, pp. 106-109, 2011.

[23] J. A. Spencer, F. Ferraro, E. Roussakis et al., "Direct measurement of local oxygen concentration in the bone marrow of live animals," Nature, vol. 508, no. 7495, pp. 269-273, 2014.

[24] T. Itkin, S. Gur-Cohen, J. A. Spencer et al., "Distinct bone marrow blood vessels differentially regulate haematopoiesis," Nature, vol. 532, no. 7599, pp. 323-328, 2016.

[25] C. R. Mantel, H. A. O’Leary, B. R. Chitteti et al., "Enhancing hematopoietic stem cell transplantation efficacy by mitigating oxygen shock," Cell, vol. 161, no. 7, pp. 1553-1565, 2015.

[26] H. E. Broxmeyer, H. A. O’Leary, X. Huang, and C. Mantel, "The importance of hypoxia and extra physiologic oxygen shock/stress for collection and processing of stem and progenitor cells to understand true physiology/pathology of these cells ex vivo," Current Opinion in Hematology, vol. 22, no. 4, pp. 273-278, 2015.

[27] D. Passaro, A. di Tullio, A. Abarrategi et al., "Increased vascular permeability in the bone marrow microenvironment contributes to disease progression and drug response in acute myeloid leukemia," Cancer Cell, vol. 32, no. 3, pp. 324341.e6, 2017.

[28] D. Duarte, E. D. Hawkins, O. Akinduro et al., "Inhibition of endosteal vascular niche remodeling rescues hematopoietic 
stem cell loss in AML," Cell Stem Cell, vol. 22, no. 1, pp. 6477.e6, 2018.

[29] A. Shinohara, Y. Imai, M. Nakagawa, T. Takahashi, M. Ichikawa, and M. Kurokawa, "Intracellular reactive oxygen species mark and influence the megakaryocyte-erythrocyte progenitor fate of common myeloid progenitors," Stem Cells, vol. 32, no. 2, pp. 548-557, 2014.

[30] R. E. Ronn, C. Guibentif, S. Saxena, and N. B. Woods, "Reactive oxygen species impair the function of CD90+ hematopoietic progenitors generated from human pluripotent stem cells," Stem Cells, vol. 35, no. 1, pp. 197-206, 2017.

[31] A. Hinge, J. Xu, J. Javier et al., "p190-B RhoGAP and intracellular cytokine signals balance hematopoietic stem and progenitor cell self-renewal and differentiation," Nature Communications, vol. 8, no. 1, 2017.

[32] A. Hirayama, K. Yoh, S. Nagase et al., "EPR imaging of reducing activity in Nrf2 transcriptional factor-deficient mice," Free Radical Biology \& Medicine, vol. 34, no. 10, pp. 1236-1242, 2003.

[33] K. Kato, K. Takahashi, S. Monzen et al., "Relationship between radiosensitivity and Nrf2 target gene expression in human hematopoietic stem cells," Radiation Research, vol. 174, no. 2, pp. 177-184, 2010.

[34] A. A. Merchant, A. Singh, W. Matsui, and S. Biswal, "The redox-sensitive transcription factor Nrf2 regulates murine hematopoietic stem cell survival independently of ROS levels," Blood, vol. 118, no. 25, pp. 6572-6579, 2011.

[35] J. J. Tsai, J. A. Dudakov, K. Takahashi et al., "Nrf2 regulates haematopoietic stem cell function," Nature Cell Biology, vol. 15, no. 3, pp. 309-316, 2013.

[36] J. H. Kim, R. K. Thimmulappa, V. Kumar et al., "NRF2-mediated Notch pathway activation enhances hematopoietic reconstitution following myelosuppressive radiation," The Journal of Clinical Investigation, vol. 124, no. 2, pp. 730-741, 2014.

[37] J. P. Chute, "NRF2 mitigates radiation-induced hematopoietic death," The Journal of Clinical Investigation, vol. 124, no. 3, pp. 960-961, 2014.

[38] S. Murakami, T. Suzuki, H. Harigae, P. H. Romeo, M. Yamamoto, and H. Motohashi, "NRF2 activation impairs quiescence and bone marrow reconstitution capacity of hematopoietic stem cells," Molecular and Cellular Biology, vol. 37, no. 19, 2017.

[39] H. Li, D. Li, Z. He et al., "The effects of Nrf2 knockout on regulation of benzene-induced mouse hematotoxicity," Toxicology and Applied Pharmacology, vol. 358, pp. 56-67, 2018.

[40] N. Kubben, W. Zhang, L. Wang et al., "Repression of the antioxidant NRF2 pathway in premature aging," Cell, vol. 165, no. 6, pp. 1361-1374, 2016.

[41] Z. Yuan, J. Zhang, Y. Huang et al., "NRF2 overexpression in mesenchymal stem cells induces stem-cell marker expression and enhances osteoblastic differentiation," Biochemical and Biophysical Research Communications, vol. 491, no. 1, pp. 228-235, 2017.

[42] H. Motohashi, M. Kimura, R. Fujita et al., "NF-E2 domination over Nrf2 promotes ROS accumulation and megakaryocytic maturation," Blood, vol. 115, no. 3, pp. 677-686, 2010.

[43] L. Zhang, D. Johnson, and J. A. Johnson, "Deletion of Nrf2 impairs functional recovery, reduces clearance of myelin debris and decreases axonal remyelination after peripheral nerve injury," Neurobiology of Disease, vol. 54, pp. 329-338, 2013.
[44] A. M. Vincent, K. Kato, L. L. McLean, M. E. Soules, and E. L. Feldman, "Sensory neurons and Schwann cells respond to oxidative stress by increasing antioxidant defense mechanisms," Antioxidants \& Redox Signaling, vol. 11, no. 3, pp. 425-438, 2009.

[45] J. J. Tsai, E. Velardi, Y. Shono et al., "Nrf2 regulates $\mathrm{CD}^{+}{ }^{+} \mathrm{T}$ cell-induced acute graft-versus-host disease in mice," Blood, vol. 132, no. 26, pp. 2763-2774, 2018.

[46] J. Jang, Y. Wang, H. S. Kim, M. A. Lalli, and K. S. Kosik, "Nrf2, a regulator of the proteasome, controls self-renewal and pluripotency in human embryonic stem cells," Stem Cells, vol. 32, no. 10, pp. 2616-2625, 2014.

[47] C. E. Hochmuth, B. Biteau, D. Bohmann, and H. Jasper, "Redox regulation by Keap1 and Nrf2 controls intestinal stem cell proliferation in_Drosophila_," Cell Stem Cell, vol. 8, no. 2, pp. 188-199, 2011.

[48] M. K. Paul, B. Bisht, D. O. Darmawan et al., "Dynamic changes in intracellular ROS levels regulate airway basal stem cell homeostasis through Nrf2-dependent Notch signaling," Cell Stem Cell, vol. 15, no. 2, pp. 199-214, 2014.

[49] I. G. Ryoo, S. H. Lee, and M. K. Kwak, "Redox modulating NRF2: a potential mediator of cancer stem cell resistance," Oxidative Medicine and Cellular Longevity, vol. 2016, Article ID 2428153, 14 pages, 2016.

[50] W. Ge, K. Zhao, X. Wang et al., "iASPP is an antioxidative factor and drives cancer growth and drug resistance by competing with Nrf2 for Keap1 binding," Cancer Cell, vol. 32, no. 5, pp. 561-573.e6, 2017.

[51] K. Ito, A. Hirao, F. Arai et al., "Regulation of oxidative stress by ATM is required for self-renewal of haematopoietic stem cells," Nature, vol. 431, no. 7011, pp. 997-1002, 2004.

[52] K. Ito, A. Hirao, F. Arai et al., "Reactive oxygen species act through p38 MAPK to limit the lifespan of hematopoietic stem cells," Nature Medicine, vol. 12, no. 4, pp. 446-451, 2006.

[53] P. Rimmelé, R. Liang, C. L. Bigarella et al., "Mitochondrial metabolism in hematopoietic stem cells requires functional FOXO3," EMBO Reports, vol. 16, no. 9, pp. 1164-1176, 2015.

[54] C. L. Bigarella, J. Li, P. Rimmelé, R. Liang, R. W. Sobol, and S. Ghaffari, "FOXO3 transcription factor is essential for protecting hematopoietic stem and progenitor cells from oxidative DNA damage," Journal of Biological Chemistry, vol. 292, no. 7, pp. 3005-3015, 2017.

[55] Z. Tothova, R. Kollipara, B. J. Huntly et al., "FoxOs are critical mediators of hematopoietic stem cell resistance to physiologic oxidative stress," Cell, vol. 128, no. 2, pp. 325-339, 2007.

[56] Z. Tothova and D. G. Gilliland, "FoxO transcription factors and stem cell homeostasis: insights from the hematopoietic system," Cell Stem Cell, vol. 1, no. 2, pp. 140-152, 2007.

[57] K. Miyamoto, K. Y. Araki, K. Naka et al., "Foxo3a is essential for maintenance of the hematopoietic stem cell pool," Cell Stem Cell, vol. 1, no. 1, pp. 101-112, 2007.

[58] V. Nogueira, Y. Park, C. C. Chen et al., "Akt determines replicative senescence and oxidative or oncogenic premature senescence and sensitizes cells to oxidative apoptosis," Cancer Cell, vol. 14, no. 6, pp. 458-470, 2008.

[59] A. Mehta, J. L. Zhao, N. Sinha et al., "The MicroRNA-132 and MicroRNA-212 cluster regulates hematopoietic stem cell maintenance and survival with age by buffering FOXO3 expression," Immunity, vol. 42, no. 6, pp. 10211032, 2015. 
[60] J. Li, W. Du, S. Maynard, P. R. Andreassen, and Q. Pang, “Oxidative stress-specific interaction between FANCD2 and FOXO3a," Blood, vol. 115, no. 8, pp. 1545-1548, 2010.

[61] X. Li, J. Li, A. Wilson, J. Sipple, J. Schick, and Q. Pang, "Fancd2 is required for nuclear retention of Foxo3a in hematopoietic stem cell maintenance," The Journal of Biological Chemistry, vol. 290, no. 5, pp. 2715-2727, 2015.

[62] P. Qian, X. C. He, A. Paulson et al., “The _Dlk1-Gtl2_ locus preserves LT-HSC function by inhibiting the PI3K-mTOR pathway to restrict mitochondrial metabolism," Cell Stem Cell, vol. 18, no. 2, pp. 214-228, 2016.

[63] Y. Liang, G. Van Zant, and S. J. Szilvassy, "Effects of aging on the homing and engraftment of murine hematopoietic stem and progenitor cells," Blood, vol. 106, no. 4, pp. 1479-1487, 2005.

[64] F. M. Rauscher, P. J. Goldschmidt-Clermont, B. H. Davis et al., "Aging, progenitor cell exhaustion, and atherosclerosis," Circulation, vol. 108, no. 4, pp. 457-463, 2003.

[65] W. W. Pang, E. A. Price, D. Sahoo et al., "Human bone marrow hematopoietic stem cells are increased in frequency and myeloid-biased with age," Proceedings of the National Academy of Sciences of the United States of America, vol. 108, no. 50, pp. 20012-20017, 2011.

[66] M. L. Porto, B. P. Rodrigues, T. N. Menezes et al., "Reactive oxygen species contribute to dysfunction of bone marrow hematopoietic stem cells in aged C57BL/6 J mice," Journal of Biomedical Science, vol. 22, no. 1, 2015.

[67] R. Khatri, S. Krishnan, S. Roy, S. Chattopadhyay, V. Kumar, and A. Mukhopadhyay, "Reactive oxygen species limit the ability of bone marrow stromal cells to support hematopoietic reconstitution in aging mice," Stem Cells and Development, vol. 25, no. 12, pp. 948-958, 2016.

[68] J. Li, D. Cai, X. Yao et al., "Protective effect of ginsenoside Rg1 on hematopoietic stem/progenitor cells through attenuating oxidative stress and the $\mathrm{Wnt} / \beta$-catenin signaling pathway in a mouse model of d-galactose-induced aging," International Journal of Molecular Sciences, vol. 17, no. 6, p. 849, 2016.

[69] X. Mu, Y. Zhang, J. Li et al., “Angelica sinensis polysaccharide prevents hematopoietic stem cells senescence in D-galactoseinduced aging mouse model," Stem Cells International, vol. 2017, Article ID 3508907, 12 pages, 2017.

[70] M. G. Poulos, P. Ramalingam, M. C. Gutkin et al., "Endothelial transplantation rejuvenates aged hematopoietic stem cell function," The Journal of Clinical Investigation, vol. 127, no. 11, pp. 4163-4178, 2017.

[71] L. Shao, Y. Luo, and D. Zhou, "Hematopoietic stem cell injury induced by ionizing radiation," Antioxidants \& Redox Signaling, vol. 20, no. 9, pp. 1447-1462, 2014.

[72] S. Rodrigues-Moreira, S. G. Moreno, G. Ghinatti et al., "Lowdose irradiation promotes persistent oxidative stress and decreases self- renewal in hematopoietic stem cells," Cell Reports, vol. 20, no. 13, pp. 3199-3211, 2017.

[73] H. Zhang, Z. Zhai, Y. Wang et al., "Resveratrol ameliorates ionizing irradiation-induced long-term hematopoietic stem cell injury in mice," Free Radical Biology \& Medicine, vol. 54, pp. 40-50, 2013.

[74] H. Wang, Y. L. Yang, H. Zhang, H. Yan, X. J. Wu, and C. Z. Zhang, "Administration of the resveratrol analogues isorhapontigenin and heyneanol-A protects mice hematopoietic cells against irradiation injuries," BioMed Research International, vol. 2014, Article ID 282657, 9 pages, 2014.
[75] G. Xu, H. Wu, J. Zhang et al., "Metformin ameliorates ionizing irradiation-induced long-term hematopoietic stem cell injury in mice," Free Radical Biology \& Medicine, vol. 87, pp. 15-25, 2015.

[76] S. Suryavanshi, D. Sharma, R. Checker et al., "Amelioration of radiation-induced hematopoietic syndrome by an antioxidant chlorophyllin through increased stem cell activity and modulation of hematopoiesis," Free Radical Biology \& Medicine, vol. 85, pp. 56-70, 2015.

[77] L. Lu, J. Dong, D. Li, J. Zhang, and S. Fan, “3,3'-diindolylmethane mitigates total body irradiation-induced hematopoietic injury in mice," Free Radical Biology \& Medicine, vol. 99, pp. 463-471, 2016.

[78] D. Li, Z. Tian, W. Tang et al., "The Protective Effects of 5Methoxytryptamine- $\alpha$-lipoic Acid on Ionizing RadiationInduced Hematopoietic Injury," International Journal of Molecular Sciences, vol. 17, no. 6, p. 935, 2016.

[79] C. Wei, H. Wen, L. Yuan et al., "Formaldehyde induces toxicity in mouse bone marrow and hematopoietic stem/progenitor cells and enhances benzene-induced adverse effects," Archives of Toxicology, vol. 91, no. 2, pp. 921-933, 2017.

[80] J. Liu, D. Y. Jia, S. Z. Cai et al., "Mitochondria defects are involved in lead-acetate-induced adult hematopoietic stem cell decline," Toxicology Letters, vol. 235, no. 1, pp. 37-44, 2015.

[81] W. Lu, M. Zhao, S. Rajbhandary et al., "Free iron catalyzes oxidative damage to hematopoietic cells/mesenchymal stem cells in vitro and suppresses hematopoiesis in iron overload patients," European Journal of Haematology, vol. 91, no. 3, pp. 249-261, 2013.

[82] X. Chai, D. Li, X. Cao et al., "ROS-mediated iron overload injures the hematopoiesis of bone marrow by damaging hematopoietic stem/progenitor cells in mice," Scientific Reports, vol. 5, no. 1, 2015.

[83] H. Shen, H. Yu, P. H. Liang et al., "An acute negative bystander effect of $\gamma$-irradiated recipients on transplanted hematopoietic stem cells," Blood, vol. 119, no. 15, pp. 3629-3637, 2012.

[84] W. Miao, R. Xufeng, M. R. Park et al., "Hematopoietic stem cell regeneration enhanced by ectopic expression of ROSdetoxifying enzymes in transplant mice," Molecular Therapy, vol. 21, no. 2, pp. 423-432, 2013.

[85] L. Hu, H. Cheng, Y. Gao et al., "Antioxidant N-acetyl-L-cysteine increases engraftment of human hematopoietic stem cells in immune-deficient mice," Blood, vol. 124, no. 20, pp. e45e48, 2014.

[86] Y. Kong, Y. Song, Y. Hu et al., "Increased reactive oxygen species and exhaustion of quiescent CD34-positive bone marrow cells may contribute to poor graft function after allotransplants," Oncotarget, vol. 7, no. 21, pp. 30892-30906, 2016.

[87] M. Benrahmoune, P. Therond, and Z. Abedinzadeh, "The reaction of superoxide radical with_N_ $\mathrm{N}_{-}$-acetylcysteine," Free Radical Biology \& Medicine, vol. 29, no. 8, pp. 775-782, 2000.

[88] P. Santus, A. Corsico, P. Solidoro, F. Braido, F. di Marco, and N. Scichilone, "Oxidative stress and respiratory system: pharmacological and clinical reappraisal of $\mathrm{N}$-acetylcysteine," COPD, vol. 11, no. 6, pp. 705-717, 2014.

[89] Y. Kong, Y. Song, F. F. Tang et al., "N-acetyl-L-cysteine improves mesenchymal stem cell function in prolonged isolated thrombocytopenia post-allotransplant," British Journal of Haematology, vol. 180, no. 6, pp. 863-878, 2018.

[90] Y. Kong, Y. Wang, Y. Y. Zhang et al., "Prophylactic oral NAC reduced poor hematopoietic reconstitution by improving 
endothelial cells after haploidentical transplantation," Blood Advances, vol. 3, no. 8, pp. 1303-1317, 2019.

[91] N. Xia, A. Daiber, U. Forstermann, and H. Li, "Antioxidant effects of resveratrol in the cardiovascular system," British Journal of Pharmacology, vol. 174, no. 12, pp. 1633-1646, 2017.

[92] N. Heinz, B. Ehrnström, A. Schambach, A. Schwarzer, U. Modlich, and B. Schiedlmeier, "Comparison of different cytokine conditions reveals resveratrol as a new molecule for ex vivo cultivation of cord blood-derived hematopoietic stem cells," Stem Cells Translational Medicine, vol. 4, no. 9, pp. 1064-1072, 2015.

[93] P. Rimmele, S. Lofek-Czubek, and S. Ghaffari, "Resveratrol increases the bone marrow hematopoietic stem and progenitor cell capacity," American Journal of Hematology, vol. 89, no. 12, pp. E235-E238, 2014.

[94] H.-J. Sim, S.-H. Kook, C.-Y. Yun, G. Bhattarai, E. S. Cho, and J. C. Lee, "Brief report: consecutive alendronate administration-mediated inhibition of osteoclasts improves long-term engraftment potential and stress resistance of HSCs," Stem Cells, vol. 34, no. 10, pp. 2601-2607, 2016.

[95] J. G. Gill, E. Piskounova, and S. J. Morrison, "Cancer, oxidative stress, and metastasis," Cold Spring Harbor Symposia on Quantitative Biology, vol. 81, pp. 163-175, 2016.

[96] N. Chen, C. Hao, B.-C. Liu et al., "Roxadustat treatment for anemia in patients undergoing long-term dialysis," New England Journal of Medicine, vol. 381, no. 11, pp. 10111022, 2019.

[97] N. Chen, C. Hao, X. Peng et al., "Roxadustat for anemia in patients with kidney disease not receiving dialysis," New England Journal of Medicine, vol. 381, no. 11, pp. 10011010, 2019. 


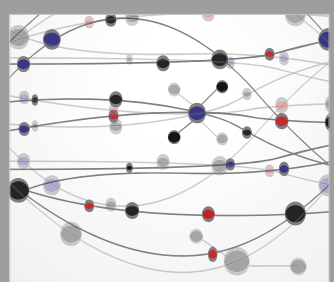

The Scientific World Journal
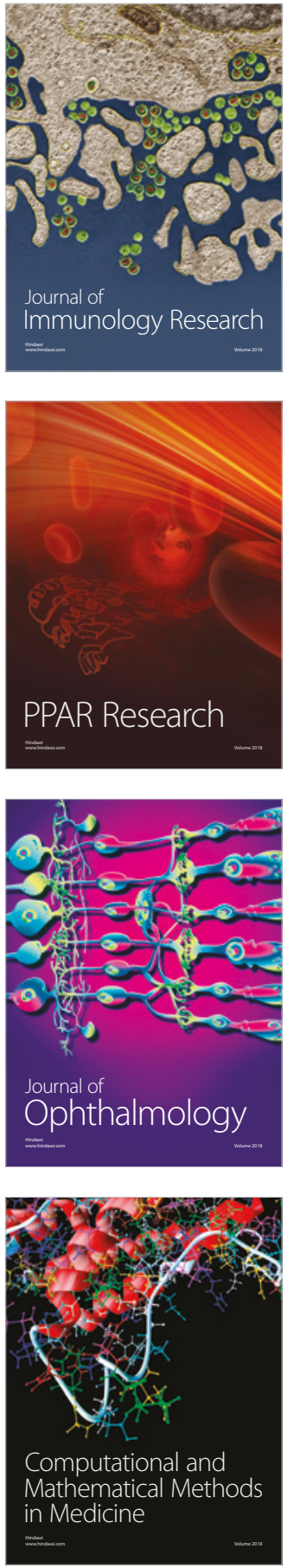

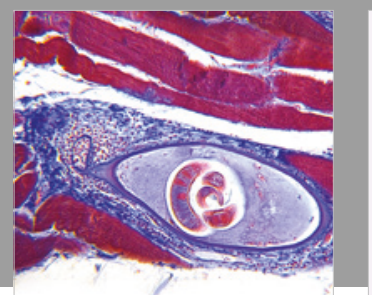

Gastroenterology Research and Practice

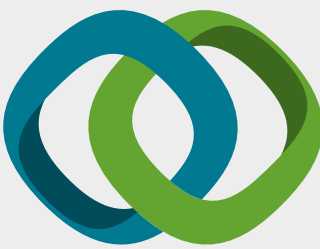

\section{Hindawi}

Submit your manuscripts at

www.hindawi.com
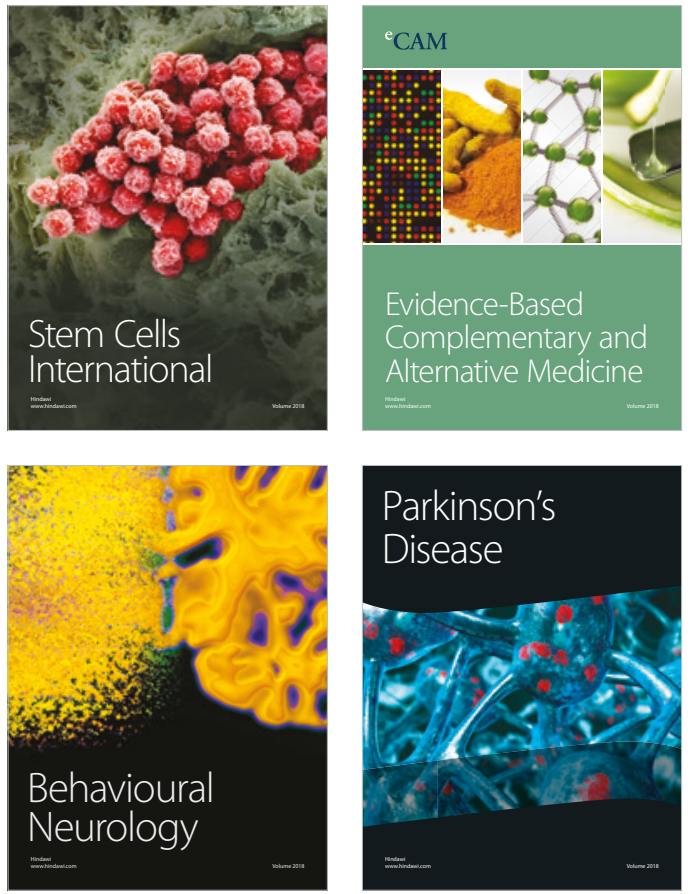

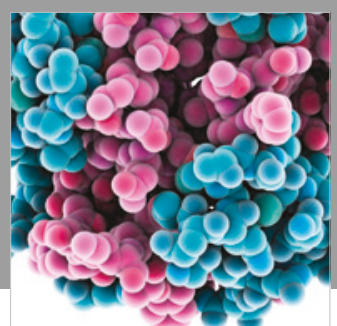

ournal of

Diabetes Research

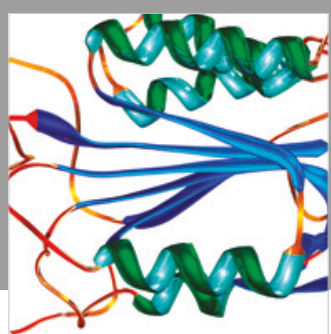

Disease Markers
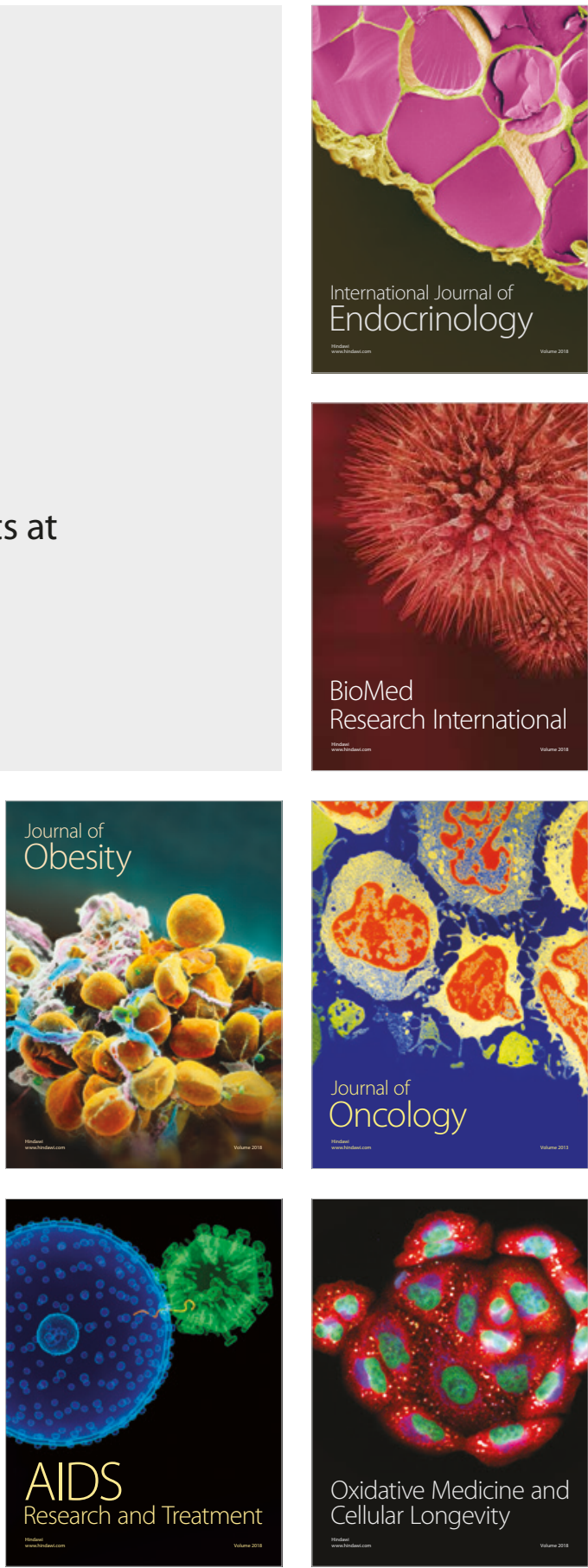